\title{
Effects of Appraisal of Sexual Stimuli on Sexual Arousal in Women with and Without Superficial Dyspareunia
}

\author{
Marieke Brauer • Moniek M. ter Kuile • Ellen Laan
}

Received: 6 November 2006/Revised: 14 November 2007 / Accepted: 19 January 2008/Published online: 17 June 2008

(C) The Author(s) 2008

\begin{abstract}
This study examined the effects of appraisal of sexual stimuli on sexual arousal in women with superficial dyspareunia $(n=50)$ and sexually functional women $(n=$ 25). To elicit different appraisals of an erotic film fragment, participants received an instruction prior to viewing it, with a focus on genital pain or on sexual enjoyment. A neutral instruction served as a control condition. Assignment to instruction condition was randomized. Genital arousal (vaginal pulse amplitude) and self-report ratings of affect and genital sensations were obtained in response to the erotic stimulus. As predicted, appraisal of the erotic stimulus affected genital responding, albeit marginally significant. Follow-up tests indicated that women who received the genital pain instruction responded with marginally significant lower genital arousal levels than women who received the sexual enjoyment instruction $(d=0.67)$. A significant instruction effect for negative affect was found, signifying that negative affect ratings were highest after the genital pain instruction and lowest after the sexual enjoyment instruction $(d=0.80)$. A marginally significant group by instruction interaction effect was observed for positive affect, indicating that women with dyspareunia reported significantly less positive affect than controls after the sexual enjoyment instruction $(d=1.48)$. Whereas women with dyspareunia reported overall marginally significant more negative affect than controls $(d=0.48)$, there were
\end{abstract}

\section{Brauer $(\bowtie) \cdot$ M. M. ter Kuile}

Department of Psychosomatic Gynecology and Sexology, Leiden

University Medical Center, Poortgebouw Zuid, Rijnsburgerweg

10, Leiden RC 2300, The Netherlands

e-mail: m.brauer@lumc.nl

\section{E. Laan}

Department of Sexology and Psychosomatic Obstetrics and Gynecology, Academic Medical Center, University of Amsterdam, Amsterdam, The Netherlands no differences in genital responsiveness between groups. These results provided preliminary evidence for the modulatory effects of appraisal of sexual stimuli on subsequent genital responding and affect in women with and without sexual complaints.

Keywords Appraisal - Dyspareunia .

Vaginal photoplethysmography $\cdot$ Sexual arousal

\section{Introduction}

Most people have expectations of enjoyment when encountering a sexual situation, although some people, under the same circumstances, experience fear, disgust, and other negative emotions. Apparently, one and the same stimulus event may be evaluated or "appraised" in different or even opposite ways. Appraisal refers to the mechanisms which give a stimulus event emotional meaning and is widely held to shape experiential/emotional, physiological, and behavioral responses to stimulus events (e.g., Cacioppo, Berntson, Larsen, Poehlmann, \& Ito, 2000). Also, current information processing models on sexual arousal (Janssen, Everaerd, Spiering, \& Janssen, 2000; Laan \& Janssen, 2007; but see also Barlow, 1986; Basson, 2002) posit that the appraisal of a sexual cue is important in determining whether or not a full-blown sexual response will occur. This means that individuals who appraise a sexual cue positively are hypothesized to maintain their attention to it. This, in turn, will further enhance autonomic arousal resulting in adequate genital sexual responding together with a subjective experience of sexual arousal. In contrast, individuals who appraise sexual stimuli in a predominantly negative way are thought to be more likely to attend to nonsexual, threatening cues (e.g., performance related concerns or anticipated pain during intercourse) which minimizes further 
processing of sexual stimuli. Distraction from sexually arousing stimuli may subsequently lead to low levels of genital and subjective sexual arousal which interferes with adequate sexual functioning (e.g., Barlow, 1986; Janssen et al., 2000; Laan \& Janssen, 2007; Sbrocco \& Barlow, 1996).

In women, lack of genital sexual arousal may lead to vaginal dryness. One of the most prevalent female sexual dysfunctions is dyspareunia (i.e., persistent or recurrent genital pain associated with intercourse), in which vaginal dryness in combination with enhanced pelvic floor muscle tone is hypothesized to play an important role in the onset and/or maintenance of this medically unexplained condition (Spano \& Lamont, 1975; ter Kuile \& Weijenborg, 2006; van Lunsen \& Ramakers, 2002).

There are some indications that a negative appraisal of sexual stimuli is implicated in dyspareunia given the observation that symptomatic women have consistently reported more negative cognitive-affective evaluations of sexual encounters than women without sexual complaints (Brauer, ter Kuile, Laan, \& Trimbos, 2008; Nunns \& Mandal, 1997; van Lankveld, Weijenborg, \& ter Kuile, 1996). Further support was obtained in an experimental study in which women with and without dyspareunia were threatened to receive painful stimuli during exposure to an erotic film fragment (Brauer, ter Kuile, Janssen, \& Laan, 2007). As compared to a control condition consisting of an erotic film without simultaneous threat, diminished genital arousal was observed in both groups of women in the threat condition. The erotic film in the threat condition also evoked more negative affect in both groups of women. These findings suggest that the threatening context in which the erotic stimulus was shown adversely influenced the meaning of that stimulus, resulting in diminished genital arousal and stronger negative affect.

Along the same lines, several experimental manipulations (e.g., suppression, performance demand, sexual scripts, stress) in sexually functional women have resulted in different levels of genital and subjective sexual arousal relative to control conditions (e.g., Kuffel \& Heiman, 2006; Laan, Everaerd, van Aanhold, \& Rebel, 1993; ter Kuile, Vigeveno, \& Laan, 2007). For instance, Beck and Baldwin (1994) asked sexually functional women to suppress sexual arousal through any means other than looking away from the screen on which an erotic film fragment was presented. Women who successfully suppressed their genital arousal responses reported the use of negative cognitive evaluations regarding the film or the sexual acts, such as "I imagined that she [the actress] was in pain." In other words, a negative (re-) appraisal of the sexual stimulus inhibited subsequent genital responding.

Corresponding with the above outlined model on sexual arousal, these cumulative findings suggest that experimental manipulations bias the way sexual stimuli are appraised, which, in turn, influences genital responses and subjective reports (Janssen et al., 2000; Laan \& Janssen, 2007).
Suggestive as these observations may be, they provide no direct support for the assumption that appraisal influences genital and subjective sexual arousal. Perhaps other cognitive (e.g., distraction) or physiological mechanisms (e.g., enhanced sympathetic nervous system activation) were (to some extent) responsible for the dissimilar levels of genital arousal in experimental versus control conditions. Despite a growing body of literature describing how appraisal mechanisms modulate physiological responses and emotional reactions to other emotional stimuli (for a review, see Ochsner \& Gross, 2005), we do not know of any direct empirical assessment of the influence of appraisal of sexual stimuli on genital and subjective sexual arousal in women. Therefore, the purpose of the present study was to experimentally manipulate directly the appraisal of a sexual stimulus in order to study its effects on sexual arousal in women with and without dyspareunia.

Relying on research in other fields of psychology that have shown that the appraisal of one and the same stimulus can be successfully manipulated by providing discrepant information regarding that stimulus (cf. Arntz \& Claassens, 2003; Lamm, Batson, \& Decety, 2007; Mosely \& Arntz, 2007), the present study used a similar methodology. All participants were presented with an erotic film fragment depicting explicit heterosexual intercourse, in which the actress was behaving ambiguously. That is, it was not obvious whether she enjoyed having intercourse or that she disliked it and maybe experienced it as painful. Prior to viewing, participants were given one of three instructions. In the genital pain instruction, participants were informed that the actress' behavior reflected expressions of genital pain. In the sexual enjoyment instruction they were told that her behavior was indicative of enjoyment and pleasure. Also a control condition was created, in which no specific information regarding the actress' behavior was given. The genital pain instruction prior to the erotic stimulus was chosen as an analogue of the expectation of experiencing pain during intercourse as assumed to exist in women with dyspareunia (e.g., Payne, Binik, Amsel, \& Khalife, 2005; Spano \& Lamont, 1975; ter Kuile \& Weijenborg, 2006), whereas the sexual enjoyment instruction was used as an analogue to the expectation of experiencing enjoyment during intercourse in sexually functional women.

In conjunction with current information processing models on sexual arousal (Janssen et al., 2000; Laan \& Janssen, 2007), it was predicted that, of all three instruction conditions, the pain instruction would result in the lowest levels of genital arousal and the sexual enjoyment instruction in the highest levels. With respect to subjective reports of affect, participants were predicted to report the highest level of negative affect and lowest level of positive affect in the genital pain instruction condition, whereas the reverse pattern was expected for the sexual enjoyment instruction condition. Based on the assumption that the modulatory effects of appraisal on sexual arousal refer to a general mechanism, we hypothesized the 
manipulation effects on genital and subjective sexual responding to point in the same direction for both women with dyspareunia and sexually functional women. Furthermore, exploratory analyses were conducted to investigate whether women with dyspareunia differed from controls in their genital arousal levels and subjective reports.

\section{Method \\ Participants}

The study sample consisted of 50 women with dyspareunia and 25 women without sexual complaints. These women had taken part in a psychophysiological study on sexual arousal and were immediately following the experimental assessment invited to stay and participate in the present study. All participants gave their informed consent for the present study and participated. The objective of the preceding study was to investigate whether women with dyspareunia and sexually functional women differed in their genital and subjective sexual arousal responses to visual sexual stimuli, including coitus (Brauer, Laan, \& ter Kuile, 2006). With respect to the present study, participants were informed that the purpose was to evaluate new erotic stimulus materials on its suitability for a future study. The Local Ethics Committee of the Leiden University Medical Center approved both studies.

The inclusion criterion for women with dyspareunia was complaints of superficial (introital) dyspareunia in minimally $50 \%$ of intercourse attempts for at least 6 months. Exclusion criteria for women with dyspareunia were somatic conditions responsible for dyspareunia (e.g., active vulvovaginal infections), generalized vulvodynia (unprovoked chronic vulvar burning, itching or irritation in the whole vulvar region), and lifelong vaginismus. Vulvar vestibulitis syndrome (VVS), recently redefined as provoked vestibulodynia (Edwards, 2004), was not an exclusion criterion since the etiology of VVS remains largely unknown (Lotery, McClure, \& Galask, 2004). Women without sexual complaints were included if they had had no sexual complaints for at least 1 year, were sexually active including intercourse, had had their first coital experience more than a year ago, and had partners without severe sexual complaints that could impede intercourse. Women from both groups were excluded if any of the following applied: pregnancy or lactation; a diagnosis of affective, psychotic or substance related disorder according to DSM-IV-TR (American Psychiatric Association, 2000); and having undergone a hysterectomy or prolapse surgery.

The screening procedure was already conducted in advance of the preceding study. Following an initial telephone screening, women underwent subsequent screening at the Sexology
Outpatient Clinic of the Department of Gynecology of a university medical center to determine further suitability for the study. Screening consisted of a structured sexual function interview, a DSM-IV-TR based semi-structured psychiatric interview (MINI International Neuropsychiatric Interview [MINI]; Lecrubier et al., 1997; Sheehan et al., 1997), and a standardized gynecological examination for sexual pain disorders (see de Kruiff, ter Kuile, Weijenborg, \& van Lankveld, 2000).

Of 113 women who responded to the advertisements, 84 (74\%) women appeared eligible during the first telephone interview and were further screened at the Sexology Outpatient Clinic. Of 57 screened women with dyspareunia, 4 (7\%) were excluded and $3(5 \%)$ eligible women withdrew from the study. Of the excluded symptomatic women, one was diagnosed with lifelong vaginismus during the physical examination and one was diagnosed with vulvodynia. The third woman experienced pain during less than $50 \%$ of intercourse attempts. The fourth woman was excluded because she experienced difficulties with comprehending the questions during the interview and with completing the questionnaires. Of the women with dyspareunia who withdrew, one explained that she was afraid of inserting the vaginal photoplethysmograph. The second woman became severely ill and the third one withdrew without offering a reason. Of 27 screened women without sexual complaints, 25 (93\%) were eligible. One ineligible woman had had first coital experience less than 1 year ago. The other woman was diagnosed with depressive disorder. Women who withdrew or were excluded after screening were paid $€ 12.50$.

At the end of the psychophysiological assessment of the preceding study, participants were presented with a 9 min neutral film clip as a return to baseline measurement. The last minute was used as the new VPA baseline level. After the participant had given her informed consent to participate in the present study, she was presented with one of the three instructions followed by the erotic film clip. Self-report ratings of affect and genital sensations were collected immediately after exposure to the erotic stimulus. Next, the credibility of the genital pain and sexual enjoyment instructions was assessed as a manipulation check. Participants received a compensatory fee of $€ 50$ for completing the entire procedure. Travelling expenses were also reimbursed.

Table 1 shows the demographic and complaint characteristics. The mean age of women with dyspareunia was 28.2 years $(S D=6.7)$ and of controls 26.6 years $(S D=7.5)$. There were no significant group differences with respect to age, relationship duration, and parental status. In contrast to controls, a significantly larger percentage of women with dyspareunia lived together with their partner. The average duration of dyspareunia was 6.2 years $(S D=4.2)$. Twenty-three $(47.9 \%)$ symptomatic women had experienced genital pain since first intercourse, and 31 (64.6\%) symptomatic women were diagnosed with VVS (provoked vestibulodynia). 
Table 1 Demographic and sexual function characteristics

Note: VVS, Vulvar Vestibulitis Syndrome; FSFI, Female Sexual Function Index

$* p<.01$

\begin{tabular}{|c|c|c|c|c|c|}
\hline \multirow[t]{2}{*}{ Characteristics } & \multicolumn{2}{|c|}{ Dyspareunia $(n=48)$} & \multicolumn{2}{|c|}{ Controls $(n=24)$} & \multirow[t]{2}{*}{$\chi^{2}$ or $t$} \\
\hline & $M$ & $S D$ & $M$ & $S D$ & \\
\hline Age (years) & 28.2 & 6.7 & 26.6 & 7.5 & $t=0.95$ \\
\hline Duration relation (years) & 6.4 & 4.8 & 4.9 & 7.3 & $t=1.05$ \\
\hline Married/co-habiting, $N(\%)$ & \multicolumn{2}{|l|}{38 (79.2) } & \multicolumn{2}{|l|}{$10(41.7)$} & $\chi^{2}=10.13^{*}$ \\
\hline Children, $N(\%)$ & \multicolumn{2}{|l|}{$5(10.4)$} & \multicolumn{2}{|l|}{$4(16.7)$} & $\chi^{2}=0.57$ \\
\hline Duration dyspareunia (years) & 6.2 & 4.2 & - & & \\
\hline \multicolumn{6}{|l|}{ Onset dyspareunia } \\
\hline Lifelong, $N(\%)$ & \multicolumn{2}{|l|}{$23(47.9)$} & \multicolumn{2}{|l|}{-} & \\
\hline Diagnosis of VVS, $N(\%)$ & \multicolumn{2}{|l|}{$31(64.6)$} & \multicolumn{2}{|l|}{-} & \\
\hline \multicolumn{6}{|l|}{ FSFI subscales } \\
\hline Desire & 2.6 & 1.1 & 4.1 & 1.0 & $t=-5.72 *$ \\
\hline Arousal & 3.1 & 1.7 & 5.1 & 1.3 & $t=-5.70^{*}$ \\
\hline Lubrication & 3.4 & 1.8 & 5.6 & 1.3 & $t=-6.16^{*}$ \\
\hline Orgasm & 3.4 & 1.9 & 4.9 & 1.6 & $t=-3.49 *$ \\
\hline Satisfaction & 3.3 & 1.3 & 5.5 & 0.5 & $t=-10.48^{*}$ \\
\hline Pain & 1.2 & 1.3 & 5.6 & 1.3 & $t=-13.68$ \\
\hline Total score & 17.1 & 6.8 & 30.9 & 5.8 & $t=-9.09^{*}$ \\
\hline
\end{tabular}

\section{Measures}

\section{Instructions}

In the experimental conditions, participants were informed that the actress' behaviors reflected either expressions of genital pain (genital pain instruction condition) or sexual enjoyment (sexual enjoyment instruction condition). In the control condition, an instruction was given in which no specific information regarding the actress' behavior was provided (neutral instruction condition). By means of a blocked randomization procedure, participants were assigned to one of the three instructions. The instruction was conveyed by the experimenter via an intercom. The genital pain instruction was: "At the moment, we are preparing another study for which we selected an erotic fragment. Up till now, several women have watched this fragment. Some of them told us that they had the impression that the actress was experiencing pain during intercourse and that she shouted because she felt pain. We would like you to watch this video as well and rate it afterwards. We are interested in your opinion." The sexual enjoyment instruction was: "At the moment, we are preparing another study for which we selected an erotic fragment. We have chosen this fragment because we had the impression that the actress really enjoyed having intercourse. We would like you to watch this video as well and rate it afterwards. We are interested in your opinion." The neutral instruction was: "At the moment, we are preparing another study for which we selected an erotic fragment. We would like you to watch this video as well and rate it afterwards. We are interested in your opinion."

\section{Stimulus Materials}

A neutral and a sexual film clip with sound were used. The neutral video clip was taken from Die Salzmänner von Tibet, a documentary about a nomadic tribe in Tibet. The duration of the neutral film clip was $9 \mathrm{~min}$. The erotic video clip was selected from One Size Fits All, a so-called women-friendly erotic film directed by Candida Royalle (Laan, Everaerd, Van Bellen, \& Hanewald, 1994). The sex stimulus was taken from the first scene of the film and lasted $3 \mathrm{~min}$. The sex clip depicted an intercourse scene with explicit penis in vagina movements. The actress in the film was behaving ambiguously: it was not obvious whether she enjoyed having intercourse or that she disliked it and maybe experienced it as painful. Although the actress encouraged the actor verbally, her nonverbal behavior suggested the opposite (e.g., cramping, pushing the actor away during initial penetration). The sounds she produced could be interpreted as either an expression of enjoyment or as an expression of pain.

\section{Manipulation Check}

In order to assure the credibility of the genital pain and sexual enjoyment instructions, two face-valid open questions were used. Participants in the genital pain instruction were asked: "Prior to watching the film fragment, we informed you that several women who have already seen this fragment thought that the female actress was experiencing pain during intercourse and that she shouted because she felt hurt. What is your opinion about the actress' behavior?" Participants in the sexual enjoyment instruction condition were asked: "We have 
selected this fragment because we had the impression that the actress really enjoyed having intercourse. What is your opinion about the actress' behavior?" Participants in the neutral condition were asked if they had any comments on the film fragment. All participants were requested to write down their answer.

\section{Genital Sexual Arousal}

To measure genital arousal, vaginal pulse amplitude (VPA) was assessed by a vaginal photoplethysmograph. The photoplethysmograph is a menstrual tampon-sized device, containing an orange-red light source and a photocell. The light source illuminates the capillary bed of the vaginal wall and the phototransistor responds to the light backscattered by the vaginal wall and the blood circulating within it. When the signal is connected to an alternating current (AC) amplifier, VPA is measured, which reflects the phasic changes in vaginal engorgement accompanying each heart beat, with larger amplitudes reflecting higher levels of vaginal vasocongestion. For a detailed description of this measure, see Laan, Everaerd, and Evers (1995). During the experimental session, VPA was continuously recorded. VPA was sampled at $20 \mathrm{~Hz}$ across baseline and subsequent trials. A two-pass algorithm for automatic artefact removal (@ Molenkamp Technical Support Group, University of Amsterdam) was used to analyze the VPA data. After artefact deletion, peak-to-trough amplitude was calculated for each remaining pulse. Depth of the probe and orientation of the light source were controlled by a plate attached to the cable within $5 \mathrm{~cm}$ of the light sensor. Participants were instructed to insert the probe such that the plate touched their labia. The probe and plate were disinfected by means of a plasma sterilization procedure between uses.

\section{Subjective Self-Report}

Immediately following the erotic film clip, the participant was asked to assess on a 7-point Likert scale the degree to which she was experiencing negative affect (six items, e.g., disgust and shame), positive (five items, e.g., excited and longing), and genital sensations (six items, e.g., genital pulsing and throbbing). The extremes of the 7-point scales were "not at all" and "very strong." These 17 items were taken from a questionnaire constructed by Heiman and Rowland (1983).

\section{Assessment of Sexual Function}

At the end of the sexological screening, participants completed the Female Sexual Function Index (FSFI; Rosen et al., 2000). The FSFI is a brief self-report measure composed of six subscales: desire, arousal, lubrication, orgasm, satisfaction, and pain. Based on a Dutch sample consisting of approximately 350 women with and without sexual complaints, the internal consistency and stability of the FSFI were found to be satisfactory to good. The FSFI's ability to discriminate between sexually functional and dysfunctional women was excellent as was the ability to predict the presence or absence of sexual complaints. Finally, the convergent and divergent construct validity was good (ter Kuile, Brauer, \& Laan, 2006).

As can be seen in Table 1, analysis of the FSFI scores indicated significantly lower levels of desire, arousal, lubrication, orgasm, and satisfaction, and significantly higher levels of pain in the dyspareunia group than the control group $(p<.01)$. These results serve as a validity check on the classification of women as sexually functional and dysfunctional.

\section{Data Analysis}

VPA data were entered in a computer program. After artefact deletion, peak-to-through-amplitude was calculated for each remaining pulse, and averaged over 30-s epochs. For the baseline recording, a mean baseline score per participant was calculated. For the erotic stimulus, a mean VPA difference score was calculated by averaging all epochs (6) of the erotic stimulus minus the mean preceding baseline.

The genital arousal data were evaluated in a 2 (Group) $\times 3$ (Instruction) ANOVA, with mean VPA difference scores as the dependent variable. In order to investigate differences in subjective experience, the three subscales, negative and positive affect, and genital sensations were evaluated in 2 (Group) $\times 3$ (Instruction) ANOVAs. For all analyses, two-tailed tests were used with $\alpha$ set at .05 . We used $f$ as an effect size measure for the ANOVA's, with $f$ being calculated as a function of $\eta^{2}$ (see Cohen, 1988, p. 284), and Cohen's $d$ for Tukey HSD post-hoc contrasts between instruction conditions. For the sake of clarity, all effect sizes were expressed as $d$ 's. For the purpose of interpretation, $d=2 f$ (see Cohen, 1988, p. 276). Cohen considered effect sizes as small $(d=.20)$, medium $(d=.50)$, and large $(d=.80)$.

Data of one participant with dyspareunia and one sexually functional woman were excluded from analyses. The data of the sexually functional woman were excluded because of too many artefacts in the assessment of VPA. The participant with dyspareunia was excluded from the analyses because the initial doubt of both the experimenter and the gynecologist about the diagnosis was further supported by inconsistencies in her answers to the questions about sexual dysfunction. Due to technical failures, both VPA data and subjective ratings of one woman with dyspareunia were lost, and only subjective ratings for another woman with dyspareunia were lost. Therefore, the VPA analysis consisted of data of 48 women with dyspareunia and 24 women without sexual complaints. The distribution of participants in the three instruction conditions was as follows: Of the 48 women with dyspareunia, 17 participants received the pain instruction, 15 the sexual enjoyment instruction, and 16 the neutral instruction. The number of women without 
sexual complaints in the three instruction conditions was 9,8 , and 7 , respectively.

Prior to analysis, all dependent variables were examined for the assumption of normality (Tabachnick \& Fidell, 2001). Data for VPA difference scores and the subscales negative and positive affect had a positively skewed distribution. For VPA, a square root transformation was applied, for negative affect a reciprocal transformation was applied, and for positive affect a logarithmic transformation.

To assess if there were any differences in baseline levels of genital responding prior to the erotic film clips between groups and conditions, the data were compared using a 2 (Group) $\times 3$ (Instruction Condition) ANOVA. It was found that VPA baselines did not significantly differ between the two groups and three instruction conditions as there were no significant main or interaction effects (all $F$ s $<1$ ).

In addition, to verify that the erotic stimulus facilitated genital responsiveness, a 2 (Group) $\times 3$ (Instruction Condition) $\times 2$ (Film Type: Neutral vs. Erotic) MANOVA was conducted with mean baseline VPA levels and mean VPA levels during the erotic clip as the dependent variables. The analysis yielded a main effect of Film Type, $F(1,66)=$ $186.73, p<.01$, indicating a significant increase in genital responses during exposure to the erotic stimulus than during the neutral stimulus. Follow-up tests indicated that, in every instruction condition, women with and without dyspareunia reacted with a significant increase in genital response to the erotic film $(p<.01)$. It thus can be concluded that the erotic film was effective in eliciting genital arousal in both groups of women during all instruction conditions.

\section{Results}

\section{Preliminary Analyses}

It is conceivable that the insertion of a vaginal probe to measure arousal elicits genital pain in women with dyspareunia whereas it may cause no discomfort in controls. If so, experiences of pain before or during the experimental manipulation, especially in the genital-pain instruction, may have affected the impact of the manipulation differently in the two groups. For instance, painful experiences may strengthen the impact of the genital pain instruction in symptomatic women. Therefore, we checked whether the insertion of the vaginal probe caused more discomfort/pain in symptomatic women than in control women. They could indicate their level of discomfort on a fivepoint Likert scale, ranging from 1 (not at all) to 5 (intensely). The mean level of discomfort in the dyspareunia group was 1.7 $(S D=0.8)$ versus $1.4(S D=0.7)$ in the control group and did not significantly differ between groups, $t(70)=1.44, p>.15)$. Pertaining to discomfort due to insertion of the probe, 24 symptomatic women (50\%) reported "not at all", $16(33.3 \%)$ "very low", and six women (12.5\%) "moderate". Only two symptomatic women reported considerable discomfort. Exclusion of their data did not change the results.

\section{Effects of the Experimental Manipulation}

\section{Genital Sexual Arousal}

Table 2 shows untransformed mean VPA responses in the three instruction conditions. A 2 (Group) $\times 3$ (Instruction) ANOVA for mean square root transformed VPA difference scores yielded a marginally significant main effect for Instruction, $F(2,66)=2.48, p=.092, d=0.54$. Participants ten$\mathrm{ded}$, as expected, to respond with lowest levels of genital sexual arousal to the erotic stimulus when it was preceded by the genital pain instruction and with highest levels of genital arousal when it was preceded by the sexual enjoyment instruction. Tukey HSD contrasts showed that genital arousal responses were only different between the genital pain instruction condition and the sexual enjoyment instruction condition $(p=.06$, $d=0.67$ ), although it was still a marginally significant effect.

\section{Subjective Self-Reports: Negative and Positive Affect, and Genital Sensations}

Table 2 shows untransformed subjective ratings. A 2 (Group) $\times$ 3 (Instruction) ANOVA was conducted with reciprocally transformed ratings of negative affect as the dependent variable. A main effect for Instruction was found, $F(2,65)=5.27, p<$ $.01, d=0.80$, indicating that, in line with predictions, women who received the genital pain instruction reported highest levels of negative affect, whereas women who received the sexual enjoyment instruction reported lowest levels of negative affect. Tukey HSD contrasts showed that the level of negative affect differed significantly between the two experimental conditions (genital pain versus sexual enjoyment) ( $p<.05, d=0.86$ ) on the one hand, and marginally significant between the sexual enjoyment instruction condition and the neutral instruction condition on the other hand $(p=.07$, $d=0.68$ ). In addition, a marginally significant main effect for Group was found, $F(1,65)=3.70, p=.059, d=0.48$, signifying that the dyspareunia group had overall marginally higher ratings of negative affect than the control group.

For ratings of (logarithmically transformed) positive affect, a 2 (Group) $\times 3$ (Instruction) ANOVA yielded a marginal interaction effect, $F(2,65)=2.98, p=.058, d=$ 0.60 . Subsequent $t$-tests were conducted to test for group differences per instruction condition. These analyses indicated that significant group differences were observed only for the sexual enjoyment instruction condition, such that the dyspareunia group reported significantly lower levels of positive affect than the control group, $t=-2.80, p<.05$, 
Table 2 Mean scores for genital sexual arousal and mean ratings on the subscales negative affect, positive affect, and genital sensations
Note: The table presents untransformed data

\begin{tabular}{|c|c|c|c|c|c|c|}
\hline \multirow[t]{2}{*}{ Condition } & \multicolumn{2}{|c|}{ Dyspareunia $(n=48)$} & \multicolumn{2}{|c|}{ Controls $(n=24)$} & \multicolumn{2}{|c|}{ Combined $(n=72)$} \\
\hline & $M$ & $S D$ & $M$ & $S D$ & $M$ & $S D$ \\
\hline \multicolumn{7}{|c|}{ Genital sexual arousal (VPA) } \\
\hline Pain & 1.17 & 0.87 & 1.34 & 0.85 & 1.23 & 0.85 \\
\hline Sexual enjoyment & 1.79 & 1.24 & 2.23 & 1.79 & 1.95 & 1.43 \\
\hline Neutral & 1.83 & 1.06 & 1.33 & 1.14 & 1.68 & 1.08 \\
\hline Combined & 1.59 & 1.08 & 1.63 & 1.32 & & \\
\hline \multicolumn{7}{|l|}{ Negative affect } \\
\hline Pain & 2.04 & 0.97 & 1.61 & 0.62 & 1.89 & 0.88 \\
\hline Sexual enjoyment & 1.52 & 0.70 & 1.08 & 0.13 & 1.36 & 0.60 \\
\hline Neutral & 1.79 & 0.91 & 1.71 & 0.64 & 1.77 & 0.82 \\
\hline Combined & 1.80 & 0.88 & 1.47 & 0.57 & & \\
\hline \multicolumn{7}{|l|}{ Positive affect } \\
\hline Pain & 2.21 & 0.81 & 2.56 & 0.94 & 2.33 & 0.86 \\
\hline Sexual enjoyment & 2.39 & 1.07 & 3.58 & 0.88 & 2.82 & 1.14 \\
\hline Neutral & 2.91 & 1.31 & 2.54 & 1.25 & 2.80 & 1.10 \\
\hline Combined & 2.50 & 1.10 & 2.89 & 1.09 & & \\
\hline \multicolumn{7}{|l|}{ Genital sensations } \\
\hline Pain & 2.64 & 0.85 & 3.32 & 1.59 & 3.00 & 1.32 \\
\hline Sexual enjoyment & 2.86 & 1.78 & 4.29 & 1.34 & 3.66 & 1.68 \\
\hline Neutral & 3.16 & 1.42 & 2.89 & 1.54 & 3.02 & 1.46 \\
\hline Combined & 2.88 & 1.36 & 3.52 & 1.58 & & \\
\hline
\end{tabular}

$d=1.24$. Next, follow-up contrasts were conducted to see how ratings of positive affect were influenced by instruction in each group separately. Analyses showed that, in the dyspareunia group, no significant differences in positive affect ratings existed between the three instruction conditions. Instruction did influence positive affect in the sexually functional group: Tukey HSD contrasts revealed that sexually functional women reported marginally significant lower positive affect when they received the pain instruction $(p=.097)$ or the neutral instruction ( $p=.081$ ) as compared to the sexual enjoyment instruction. Women did not differ in their ratings of positive affect in the genital pain and neutral instruction condition. Finally, a 2 (Group) $\times 3$ (Instruction) ANOVA with ratings of genital sensations as the dependent variable revealed no significant main or interaction effects.

\section{Manipulation Check}

With respect to the genital pain instruction, 15 (88\%) out of 17 women with dyspareunia answered that they thought that the actress experienced pain during intercourse against 2 (22\%) out of nine control women. A subsequent chi-square test revealed that the percentage of women who thought that the actress experienced pain during intercourse was significantly higher in the dyspareunia group than in the control group, $\chi^{2}(1)=11.33, p<.01$. This finding indicates that the genital pain instruction was especially credible for symptomatic women. With respect to the sexual enjoyment condition, the majority of women answered to the question "What is your opinion about the actress' behavior?" with statements signifying that the actress had exaggerated her expressions of enjoyment. It was impossible to determine whether these statements indicated that the instruction was credible or not.

\section{Correlations Between Genital Sexual Arousal and Subjective Self-Reports}

Pearson product-moment correlations were calculated between mean VPA difference scores and mean scores for ratings of genital sensations, positive and negative affect collapsed across instruction conditions and groups. Only a significant and positive correlation was found between VPA levels and genital sensations, $r(69)=.27, p<.05$.

\section{Discussion}

This study was the first to investigate directly the effects of experimentally manipulated appraisal of a sexual stimulus on sexual arousal in women with and without dyspareunia. The main findings can be summarized as follows: (1) Appraisal of a sexual stimulus (erotic film fragment) appeared to modulate genital arousal responses and evaluations of negative affect. (2) Whereas women with dyspareunia did not differ in their genital responsiveness from sexually functional women, they 
reported overall higher levels of negative affect to the erotic stimulus. (3) The sexually functional women seemed more susceptible to the appraisal manipulations given that the largest differences in positive affect resulting from the instructions were found in this group.

The experimental manipulation of appraisal of a sexual stimulus appeared to evoke the intended patterns of genital arousal levels. That is, women who received negative information concerning the erotic film fragment (focus on genital pain) prior to viewing the fragment tended to respond with lower levels of genital arousal as compared to women who received positive information (focus on sexual enjoyment). These results concur with the findings obtained by Beck and Baldwin (1994) in their study on instructional control of genital responding in sexually functional women. They found that women who successfully suppressed their levels of genital arousal reported the use of negative cognitive evaluations regarding the film or the sexual acts. Moreover, the present results corroborate the modulatory effects of appraisal on physiological responses to other emotional stimuli as reported in earlier studies (see, e.g., Ochsner \& Gross, 2005).

Although genital arousal levels in the control condition (neutral information) were, as expected, in between the levels in the two experimental conditions, the effect of the information about the sexual stimulus on genital responsiveness was only marginally significant when the three instruction conditions were statistically compared. Unfortunately, followup contrasts revealed that differences in genital responding between the two experimental conditions were also marginally significant $(p=.06)$. However, the effect size $(d=0.67)$ that was obtained, points to the magnitude of the impact of appraisal on subsequent genital arousal in the two experimental conditions. As the medium effect size indicates that the sample sizes in the present study were too small, it therefore is conceivable that the study was not sufficiently powered to substantiate these differences. Taken into consideration this medium effect size, an $\alpha$-value of .05, and a power of .80, only a larger sample size -at least 36 participants per cell- would find a significant differential effect of appraisal of the sexual stimulus on genital arousal responses. Following this, it would be advisable to replicate the present study with a larger sample size in order to arrive at a more solid conclusion regarding the differential impact of appraisal of sexual stimuli on genital responding.

Both women with and without dyspareunia tended to respond with lower levels of genital arousal to the genital pain instruction in contrast to the sexual enjoyment instruction. This observation supports our hypothesis that the modulatory effects of appraisal on sexual arousal refer to a general mechanism in women, irrespective of sexual function status.

Exploratory analysis revealed that the dyspareunia group did not differ in their genital responsiveness from controls. The absence of differences in genital arousal between women with and without dyspareunia concurs with previous laboratory research showing that, relative to controls, women with dyspareunia (Brauer et al., 2006; Brauer et al., 2007; Payne et al., 2007 ) and also women with sexual arousal dysfunction (e.g., Brotto, Basson, \& Gorzalka, 2006; Laan, van Driel, \& van Lunsen, 2008; Meston \& Gorzalka, 1996; Middleton, Kuffel, \& Heiman, 2008; Morokoff \& Heiman, 1980; Salemink \& van Lankveld, 2006) were equally genitally responsive. These cumulative findings point to a lack of physiological differences between women with and without superficial dyspareunia, at least under laboratory conditions.

The experimental manipulation of appraisal of a sexual stimulus also had the intended impact on ratings of negative affect such that women who received the genital pain instruction reported significantly more negative affect than women who received the sexual enjoyment instruction. In addition, the dyspareunia group reported overall marginally significantly more negative affect in response to the erotic stimulus than the control group, with a medium effect size $(d=0.48)$. This latter finding parallels those of previous research in women with dyspareunia as well as other female sexual dysfunction groups (e.g., Brauer et al., 2007; Brauer, de Jong, Huijding, Laan, \& ter Kuile, 2008; Laan et al., 2008; Meston, 2006; Morokoff \& Heiman, 1980), demonstrating that dysfunctional women report more negative affect to sexual stimuli in general. Elevated reports of negative affect in women with dyspareunia have not only been restricted to the laboratory context, but have also been observed in the home situation (Brauer, ter Kuile et al., 2008; Nunns \& Mandal, 1997; van Lankveld et al., 1996). These cumulative findings point to a negative appraisal of sexual stimuli associated with sexual dysfunction in women.

The impact of appraisal of a sexual stimulus on ratings of positive affect seemed to be especially apparent in the sexually functional group. These women reported significantly higher levels of positive affect when they received the sexual enjoyment instruction than when they received the genital pain instruction and neutral instruction, respectively. Relative to controls, women with dyspareunia reported significantly less positive affect in the sexual enjoyment instruction condition. Since the same sample of symptomatic women also experienced less positive affect during exposure to sexual stimuli in the experimental study that was conducted prior to the present one (Brauer et al., 2006), this would suggest that women with dyspareunia report less positive affect to sexual stimuli in general. Again, it is possible that the study was not sufficiently powered to substantiate the differences between the two groups in the genital pain instruction condition.

All in all, the current research provided a unique experimental exploration of the effects of appraisal of sexual stimuli on subsequent sexual arousal in both sexually functional and dysfunctional women. Based on the reasoning that instructions regarding a sexual stimulus influence the appraisal of that 
stimulus, the present results provide preliminary support for current information processing models on sexual arousal to the extent that appraisal is assumed to modulate levels of genital and subjective sexual arousal (Janssen et al., 2000; Laan \& Janssen, 2007). These models presume that when a stimulus elicits predominantly sexual meaning in the memory system, attention further enhances the processing of sexual meaning, eventually leading to the occurrence of a full-blown activation of genital as well as subjective arousal responses. In contrast, inhibition of genital and subjective sexual responding can be understood to involve a predominantly non-sexual or emotionally negative appraisal of relevant stimuli. The sexual enjoyment instruction in the present study might have elicited a positive meaning (appraisal) of the erotic film fragment, leading to enhanced genital arousal responses and reduced levels of negative affect, whereas the instruction with a focus on genital pain might have provoked a predominantly negative appraisal of the sexual stimulus, leading to impaired genital responding and attenuated negative affect.

The observation that the erotic stimulus elicited genital arousal responses after the genital pain instruction in comparison to baseline corresponds with previous studies demonstrating that in women an automatized genital response to sexual stimuli occurs, even when these stimuli have a threatening content (e.g., Both, Everaerd, \& Laan, 2003; Laan et al., 1995;) or are presented in a threatening context (e.g., Brauer et al., 2007; ter Kuile et al., 2007). These cumulative findings can be explained in terms of the above information processing model on sexual arousal (Janssen et al., 2000; Laan \& Janssen, 2007) which posit that different levels of cognitive processing differentially influence sexual arousal responses. More precisely, when exposed to a sexual cue, the initial -unconscious- appraisal of that cue automatically elicits a genital response. As a next step, the conscious appraisal of the stimulus as emotional (e.g., "this is [not] a sexual arousing stimulus") further determines whether or not a full-blown genital response will occur together with a subjective experience of sexual arousal. Thus, whereas exposure to the erotic stimulus immediately elicited genital responses, the genital pain instruction might have biased the conscious appraisal of the erotic film fragment, such that further genital responding was impaired.

Although women with dyspareunia and controls reacted genitally similarly under the same laboratory conditions, we speculate that differences in genital responding between these groups of women may become apparent in the more naturalistic situation at home. We expect that the control group only appraised the sexual stimulus as negative in the laboratory setting, because they were influenced by negative information regarding painful intercourse prior to watching the erotic stimulus. It is assumed that they normally appraise sexual stimuli in a positive way, based on past positive sexual experiences. Conversely, in symptomatic women, it is hypothesized that their memories contain more, or more easily accessible threat- or fearful-related information based on prior sexual experiences (e.g., Barlow, 1986; Everaerd, Both, \& Laan, 2006; Janssen et al., 2000; van der Velde, \& Everaerd, 2001). Hence, they may appraise daily life sexual situations in a predominantly non-sexual, negative way (e.g., "intercourse is painful"). In this respect it is interesting to note that the majority of symptomatic women, but only a minority of control women, concurred with the suggestion in the genital pain condition that the actress was experiencing pain. This suggests that symptomatic women more readily associate a sexual situation with pain. In conclusion, our study suggests that appraisal of a sexual situation is an important determinant in subsequent genital responding and experienced affect in women, irrespective of their sexual function status.

Acknowledgements The authors would like to thank Philomeen Weijenborg, M.D., and Corrie Vliet Vlieland, M.D., for performing the medical screenings; and Bert Molenkamp and Cor Kramer for technical support. Thanks are expressed to Stephanie Both for her valuable comments on an earlier draft of this article. The authors would also thank Prof. Gert van Dijk for offering the possibility to perform this research at the Department of Neurology.

Open Access This article is distributed under the terms of the Creative Commons Attribution Noncommercial License which permits any noncommercial use, distribution, and reproduction in any medium, provided the original author(s) and source are credited.

\section{References}

American Psychiatric Association. (2000). Diagnostic and statistical manual of mental disorders (Fourth ed., text rev.). Washington, DC: Author.

Arntz, A., \& Claassens, L. (2003). The meaning of pain influences its experienced intensity. Pain, 109, 20-25.

Barlow, D. H. (1986). The causes of sexual dysfunction: The role of anxiety and cognitive interference. Journal of Consulting and Clinical Psychology, 54, 140-148.

Basson, R. (2002). A model of women's sexual arousal. Journal of Sex and Marital Therapy, 28, 1-10.

Beck, J. G., \& Baldwin, L. E. (1994). Instructional control of female sexual responding. Archives of Sexual Behavior, 23, 665-684.

Both, S., Everaerd, W., \& Laan, E. (2003). Modulation of spinal reflexes by aversive and sexually appetitive stimuli. Psychophysiology, 40, 174-183.

Brauer, M., de Jong, P. J., Huijding, J., Laan, E., \& ter Kuile, M. M. (2008). Automatic and deliberate affective associations with sexual stimuli in women with superficial dyspareunia. Archives of Sexual Behavior. doi: 10.1007/s10508-008-9367-4.

Brauer, M., Laan, E., \& ter Kuile, M. M. (2006). Sexual arousal in superficial dyspareunia. Archives of Sexual Behavior, 35, 187-196.

Brauer, M., ter Kuile, M. M., Janssen, S. A., \& Laan, E. (2007). The effect of pain-related fear on sexual arousal in women with superficial dyspareunia. European Journal of Pain, 11, 788-798.

Brauer, M., ter Kuile, M. M., Laan, E., \& Trimbos, B. (2008). Cognitive-affective correlates and predictors of superficial dyspareunia. Journal of Sex and Marital Therapy, in press.

Brotto, L. A., Basson, R., \& Gorzalka, B. B. (2006). Psychophysiological assessment in premenopausal arousal disorder. Journal of Sexual Medicine, 1, 266-277. 
Cacioppo, J. T., Berntson, G. G., Larsen, J. T., Poehlmann, K. M., \& Ito, T. A. (2000). The psychophysiology of emotion. In R. Lewis \& J. M. Haviland-Jones (Eds.), Handbook of emotion (pp. 173191). New York: Guilford Press.

Cohen, J. (1988). Statistical power analysis for the behavioral science (2nd ed.). Hillsdale, NJ: Lawrence Erlbaum Associates.

de Kruiff, M. E., ter Kuile, M. M., Weijenborg, P. T. M., \& van Lankveld, J. J. D. M. (2000). Vaginismus and dyspareunia: Is there a difference in clinical presentation? Journal of Psychosomatic Obstetrics and Gynaecology, 21, 149-155.

Edwards, L. (2004). Subsets of vulvodynia-overlapping characteristics. Journal of Reproductive Medicine, 49, 883-887.

Everaerd, W., Both, S., \& Laan, E. (2006). The experience of sexual emotions. Annual Review of Sex Research, 17, 183-199.

Heiman, J. R., \& Rowland, D. L. (1983). Affective and physiological sexual response patterns: The effect of instructions on sexually functional and dysfunctional men. Journal of Psychosomatic Research, 27, 105-116.

Janssen, E., Everaerd, W., Spiering, M., \& Janssen, J. (2000). Automatic processes and the appraisal of sexual stimuli: Toward an information processing model of sexual arousal. Journal of Sex Research, 37, 8-23.

Kuffel, S. W., \& Heiman, J. R. (2006). Effects of depressive symptoms and experimentally adopted schemas on sexual arousal and affect in sexually healthy women. Archives of Sexual Behavior, 35, 160-174.

Laan, E., Everaerd, W., \& Evers, A. (1995). Assessment of female sexual arousal: Response specifity and construct validity. Psychophysiology, 32, 376-485.

Laan, E., Everaerd, W., van Aanhold, \& Rebel, M. (1993). Performance demand and sexual arousal in women. Behaviour Research and Therapy, 31, 25-35.

Laan, E., Everaerd, W., van Bellen, G., \& Hanewald, G. (1994). Women's sexual and emotional responses to male- and femaleproduced erotica. Archives of Sexual Behavior, 23, 153-170.

Laan, E., \& Janssen, E. (2007). How do men and women feel? Determinants of subjective experience of sexual arousal. In E. Janssen (Ed.), The psychophysiology of sex (pp. 278-290). Bloomington: Indiana University Press.

Laan, E., van Driel, E., \& van Lunsen, R. (2008). Genital responsiveness in healthy women with and without sexual arousal disorder. Journal of Sexual Medicine, in press.

Lamm, C., Batson, C. D., \& Decety, J. (2007). The neural substrate of human empathy: Effects of perspective-taking and cognitive appraisal. Journal of Cognitive Neuroscience, 19, 42-58.

Lecrubier, Y., Sheehan, D. V., Weiller, E., Amorim, P., Bonora, I., Sheehan, K. H., et al. (1997). The Mini International Neuropsychiatric Interview (MINI). A short diagnostic structured interview: Reliability and validity according to the CIDI. European Psychiatry, 12, 224-231.

Lotery, H. E., McClure, N., \& Galask, R. P. (2004). Vulvodynia. Lancet, 363, 1058-1060.

Meston, C. M. (2006). The effects of state and trait self-focused attention on sexual arousal in sexually functional and dysfunctional women. Behaviour Research and Therapy, 44, 515-532.

Meston, C. M., \& Gorzalka, B. B. (1996). Differential effects of sympathetic activation on sexual arousal in sexually dysfunctional and functional women. Journal of Abnormal Psychology, 105, 582-591.

Middleton, L. S., Kuffel, S. W., \& Heiman, J. R. (2008). Effects of experimentally adopted sexual schemas on vaginal response and subjective sexual arousal: A comparison between women with sexual arousal disorder and sexually healthy women. Archives of Sexual Behavior, 37. doi: 10.1007/s10508-007-9310-0.
Morokoff, P., \& Heiman, J. (1980). Effects of erotic stimuli on sexually functional and dysfunctional women: Multiple measures before and after sex therapy. Behaviour Research and Therapy, 18, 127-137.

Moseley, G. L., \& Arntz, A. (2007). The context of a noxious stimulus affects the pain it evokes. Pain, 133, 64-71.

Nunns, D., \& Mandal, D. (1997). Psychological and psychosexual aspects of vulvar vestibulitis. Genitourinary Medicine, 73, 541-544.

Ochsner, K. N., \& Gross, J. J. (2005). The cognitive control of emotion. Trends in Cognitive Sciences, 9, 242-249.

Payne, K. A., Binik, Y. M., Amsel, R., \& Khalifé, S. (2005). When sex hurts, anxiety and fear orient attention towards pain. European Journal of Pain, 9, 427-436.

Payne, K. A., Binik, Y. M., Pukall, C. F., Thaler, L., Amsel, R., \& Khalifé, S. (2007). Becoming sexually aroused is a sensitive issue: The influence of sexual arousal on genital and non-genital sensation in women. Archives of Sexual Behavior, 36, 289-300.

Rosen, R., Brown, C., Heiman, J., Leiblum, S., Meston, C., Shabsigh, R., et al. (2000). The Female Sexual Function Index (FSFI): A multidimensional self-report instrument for the assessment of female sexual function. Journal of Sex and Marital Therapy, 26, 191-208.

Royalle, C. (Producer). (2000). One size fits all [Motion Picture]. (Available from Private Benelux BV, Best).

Salemink, E., \& van Lankveld, J. J. D. M. (2006). The effects of increasing neutral distraction on sexual responding of women with and without sexual problems. Archives of Sexual Behavior, 35, 179-190.

Sbrocco, T., \& Barlow, D. H. (1996). Conceptualizing the cognitive component of sexual arousal: Implications for sexuality research and treatment. In P. M. Salkovskis (Ed.), Frontiers of cognitive therapy (pp. 419-449). New York: Guilford Press.

Sheehan, D. V., Lecrubier, Y., Sheehan, K. H., Janavs, J., Weiller, E., Keskiner, A., et al. (1997). The validity of the Mini International Neuropsychiatric Interview (MINI) according to the SCID-P and its reliability. European Psychiatry, 12, 232-241.

Spano, L., \& Lamont, J. A. (1975). Dyspareunia: A symptom of female sexual dysfunction. The Canadian Nurse, 8, 22-25.

Tabachnick, B. G., \& Fidell, L. S. (2001). Using multivariate statistics (4th ed.). Boston: Allyn \& Bacon.

ter Kuile, M. M., Brauer, M., \& Laan, E. (2006). The Female Sexual Function Index (FSFI) and the Female Sexual Distress Scale (FSDS): Psychometric properties within a Dutch population. Journal of Sex and Marital Therapy, 32, 289-304.

ter Kuile, M. M., Vigeveno, D., \& Laan, E. (2007). Preliminary evidence that acute and chronic daily psychological stress affect sexual arousal in sexually functional women. Behaviour Research and Therapy, 45, 2078-2089.

ter Kuile, M. M., \& Weijenborg, P. T. M. (2006). A cognitive-behavioral group program for women with vulvar vestibulitis syndrome (VVS): Factors associated with treatment success. Journal of Sex and Marital Therapy, 32, 199-213.

van der Velde, J., \& Everaerd, W. (2001). The relationship between involuntary pelvic floor muscle activity, muscle awareness, and experienced threat in women with and without vaginismus. Behaviour Research and Therapy, 39, 395-408.

van Lankveld, J. J. D. M., Weijenborg, P. T. M., \& ter Kuile, M. M., (1996). Psychologic profiles of and sexual function in women with vulvar vestibulitis and their partners. Obstetrics and Gynecology, $88,65-69$.

van Lunsen, H. W., \& Ramakers, M. (2002). The hyperactive pelvic floor syndrome (HPFS): Psychosomatic and psycho-sexual aspects of hyperactive pelvic floor disorders with comorbidity of urogynecological, gastrointestinal and sexual symptomatology. Acta Endoscopia, 32, 275-285. 